\section{Mascara on the face of the Earth}

\section{Andrew Hill}

The Pleistocene: Geology and Life in the Quaternary Ice Age.

By Tage Nilsson.

Reidel/MTP Press: 1983. Pp.650. Dfl.265, $\$ 115$, £67.25.

MY former professor of geology has been known to refer to the Pleistocene as "a geological cosmetic", a superficial and possibly deceptive covering to the meat and bones beneath. Yet although study of the deeper anatomy forms the bulk of geologists' work, there is much to be said for the surface smear. Even if the rocks of this period are in a sense superficial geologically, from the point of view of life they are more fundamental - preserving important evidence about the establishment of present-day plants and animals, and recording the increasing involvement of human beings with the ecosystem.

The Quaternary is a good instance of the historical legacy of a discipline controlling to a great extent the way evidence is collected and viewed. Penck and Bruckner's scheme of glacials and interglacials, based on the Danube, was gradually extended to a Eurocentric conception of the whole world. Work in the past few years has led to a greater appreciation of the complexity of the situation and a realization of the inadequacies of the simple traditional interpretation. The new awareness has grown principally from extensive deep-sea investigations - a part of the Earth less encumbered by classical stratigraphy and notions - but also from a more detailed examination of terrestrial evidence in Europe and elsewhere. Previously there was a tendency to believe that naming constituted understanding and knowledge, whereas more stress is now placed upon process and on the interrelationships between different classes of data.

Tage Nilsson's The Pleistocene, a translation and extensive revision of an earlier Swedish textbook, reflects the conventional taxonomic approach. In some ways it may be regarded as a narrative dictionary, which is not to suggest that it is

\section{New in paperback}

- The Self and its Brain: An Argument for Interactionism by Karl R. Popper and John C. Eccles (issued by Routledge \& Kegan Paul, price $£ 7.95$, \$14.95). The book was originally published by Springer-Verlag in 1977, and was reviewed in Nature 272, 770; 1978.

- Scientific Temperaments: Three Lives in Contemporary Science by Philip J. Hilts (issued by Touchstone, an imprint of Simon \& Schuster, New York, price \$7.95). For review see Nature 302, 767; 1983. dull. On the contrary, it is an account which although terse is readable and consistently informative. Beginning with a discussion of basic concepts, the Tertiary background and developments leading to the Ice Age, and lower boundary problems, Nilsson goes on to deal with the key areas of the Alps and Italy, the British Isles and the rest of Europe, before describing other continents and the oceans. Where appropriate, each section includes a treatment of the mammalian faunas and human fossils. The sections on human palaeontology and archaeology, while accurate, are traditional and not particularly profound. There are systematic reviews of the Quaternary mammals and a classification of the vascular plants that are discussed. The book is also traditional in the sense that it has the usual European emphasis. About 250 pages are devoted to Europe, whereas Africa merits only 23, and Asia only 25. Admittedly these areas are less well documented than Europe, but even North America, where much work has been carried out, is represented by only 50 pages.

Nilsson's objects are to provide an account of progress in research and of

\section{Better spectroscopy}

\section{D.W. Turner}

\section{Introduction to Photoelectron}

Spectroscopy.

By Pradip K. Ghosh.

Wiley: 1983. Pp.377. £52.25, \$55.

AN EARLIER volume by Pradip Ghosh, $A$ Whiff of Photoelectron Spectroscopy (Wiley, 1978), proved to be an interesting and stimulating book both for experts in the field and for those about to enter it. Though somewhat idiosyncratic in style, it was obviously the work of an enthusiast. Perhaps the present volume is to be seen both as an introduction to what is now a very broad subject and a standard research text. The author has had the opportunity to build upon his earlier volume, expanding the areas where coverage was less than full and improving the order of presentation.

After an introductory chapter, there is an account of experimental techniques which occupies some $10 \%$ of the book. It has to be said, however, that this is perhaps the least critically written chapter and does not compare with the thoroughness of some of the later sections, notably the more theoretically inclined ones. Separate chapters then deal with the principal phenomena observed in core and valence shell photoelectron spectra. The first of these topics is the more systematically treated, proceeding through core binding energies and chemical shift theories (with useful comparisons with NMR and Mossbauer chemical shifts), and a smal selection of illustrative applications, to brief @)1984 Nature Publishing Group current problems, and a guide to the literature, the emphasis being on the stratigraphical and palaeontological aspects including the evolution of human beings and of culture. In this he largely succeeds. It would be good to have the same data integrated more analytically around questions of process and interrelationships, and in terms of hypotheses about geological, biological and climatic change; that, however, would be a different book.

As it is, The Pleistocene provides a good historical background, is generally up to date and contains a comprehensive bibliography of over 2,000 items. And while the illustrations sometimes serve more the needs of ornamentation than of instruction, there are a lot of them. In a text of this generality and scope specialists are bound to find points of fact or emphasis to quibble with, but taken as a whole it is an impressive achievement that should be of great value to the many geologists, biologists and other scientists who are held in fascination by the intriguing mascara on the face of the Earth.

Andrew Hill is a Research Fellow at Harvard University.

mention of shake-up and shake-off processes and collective resonances.

The phenomena observed in valence photoelectron spectra are so much more complex, however, that a comparable systematic treatment has not proved possible, and instead the author has picked out some of the main areas of interest from the literature of the past 25 years. Unfortunately his summary is really quite uncritical. In consequence this is a tantalizing, stimulating and sometimes frustrating section, but one which an advanced chemistry student with a well-developed critical faculty could well be advised to read.

The second half of the book divides between discussion of application to solid surfaces and a consideration of some of the more fundamental aspects of photoionization. One of the most useful sections is that containing the references and notes, which are collected together at the end of the book, organized by chapter and topic, though these occasionally appear to be afterthoughts which could more usefully have been included in the text.

Altogether this is a useful and helpful text. It is a considerable improvement on its predecessor, but not sufficiently systematic to be treated as a standard work on the subject since there are a number of important topics which are simply omitted or only cited briefly; for example, JahnTeller and Renner-Teller effects, which are particularly important in valence shell photoelectron spectroscopy, are mentioned only in passing and not explained.

D.W. Turner is Reader at the Physical Chemistry Laboratory, University of Oxford. 